\title{
Mutación BRAF V600E en pacientes con cáncer de tiroides. Fundación Clínica Valle del Lili: una serie de casos
}

\author{
Guillermo Edinson Guzmán ${ }^{1}$, Luz Ángela Casas ${ }^{1}$, Julián David Orrego Celestino², Juliana \\ Escobar ${ }^{3}$, Lisa Rodriguez $z^{4}$, Veline Martinez ${ }^{5}$
}

${ }^{1}$ Médico Internista Endocrinólogo, Profesor Clínico asociado Universidad ICESI, Fundación Clínica Valle del Lili

${ }^{2}$ Médico Internista, Universidad del Valle

${ }^{3}$ Médico Patóloga, PhD, Profesor asociado Universidad ICESI

Fundación Clínica Valle del Lili

${ }^{4}$ Médico Genetista, MSD, PhD, Fundación Clínica Valle del Lili

${ }^{5}$ Médico Internista, Profesor Clínico asociado Universidad ICESI, Fundación Clínica Valle del Lili

Correspondencia: Guillermo Edinson Guzmán.

Correo electrónico: guillermoeguzman@gmail.com

Fuente de financiación y conflicto de intereses: Ninguno

Fecha de recepción: 26/06/2016

Fecha de aceptación: 15/07/2016

\section{Resumen}

Objetivo: Describir las características clínicas y los hallazgos histopatológicos de los pacientes con diagnóstico de cáncer de tiroides y estudio de la mutación del Gen BRAF V600E.

Métodos: Estudio descriptivo, retrospectivo, con información obtenida de las historias clínicas de los pacientes con diagnóstico de cáncer de tiroides atendidos durante 2014 y 2105 en la Fundación Clínica Valle del Lili con estudio para la mutación del gen BRAF V600E.

Resultados: De los 344 pacientes con diagnóstico de cáncer de tiroides durante los años 2014 y 2015, se les realizó estudio de la mutación BRAF V600E a 24. La edad promedio fue de 47 años, con predominio en mujeres (87,5\%), fueron positivos para la mutación $66 \%$ de los pacientes. En relación a las características histopatológicas, el 95,8\% de los casos correspondían a cáncer papilar de tiroides, la mayoría de la variedad clásica. Los pacientes con la mutación BRAF V600E tenían mayor extensión extratiroidea, invasión linfática, invasión vascular y compromiso ganglionar, pero no se encontró relación con respecto a tamaño tumoral, multicentralidad, bilateralidad, tiroiditis de Hashimoto o presencia de metástasis.

Conclusión: Este es el primer estudio en Colombia, que describe las características clínicas e histopatológicas de los pacientes con cáncer de tiroides en relación a la presencia de la mutacion del Gen BRAF.

\section{Abstract}

Objective: To describe the clinical and histopathological findings of patients diagnosed with thyroid cancer and BRAF V600E gene mutation study.

Methods: A descriptive, retrospective study, with information obtained from the medical records of patients diagnosed with thyroid cancer seen during 2014 and 2105 in the Fundacion Clínica Valle del Lili with analysis of the BRAF V600E gene mutation.

Results: Of the 344 patients diagnosed with thyroid cancer during the years 2014 and 2015, underwent study of the BRAF V600E to 24. The average age was 47 years, with prevalence in women (87.5\%) were positive for mutation $66 \%$ of patients. Regarding the histopathologic features, $95.8 \%$ of the cases were papillary thyroid cancer, most classic variety. Patients with BRAF V600E mutation were more extrathyroid extension, lymphatic invasion, vascular invasion and nodal involvement, but no relationship was found with respect to tumor size, multicentrality, bilateralism, Hashimoto's thyroiditis or presence of metastasis.

Conclusion: This is the first study in Colombia, describing the clinical and histopathologic of patients with thyroid cancer in relation to the presence of the BRAF gene mutation characteristics.

\section{Introducción}

El cáncer de tiroides es la neoplasia endocrina más frecuente en el mundo, su incidencia se ha incrementado rápidamente a nivel mundial ${ }^{(1)}$. En Colombia, según datos del Instituto Nacional de Cancerología, la incidencia calculada para el 2011 en mujeres fue de 9,7 por 100.000 habitantes y en hombres de 1,3 por 100.000 habitantes $^{(2)}$. En el mundo, más del $90 \%$ de los casos de cáncer de tiroides corresponden a carcinoma diferenciado de tiroides (CDT), aproximadamente el 88\% corresponde a carcinoma papilar y una menor proporción a cáncer folicular.

Esta neoplasia tiene alto índice de curación con una tasa de supervivencia alta, mayor del 90\%; sin embargo, hay un importante grupo de pacientes que fallecen por esta enfermedad. Existen factores clínicos que se han relacionado con mal 
pronóstico, como: la edad avanzada ( $>45$ años), el tamaño del tumor (mayores de $5 \mathrm{~cm}$ ), la presencia de extensión extratiroidea, tumores multifocales y la metástasis a ganglios linfáticos ${ }^{(3)}$.

El gen BRAF es el encargado de producir una proteína conocida como B-RAF, que hace parte de la familia de la RAF cinasa, complejo proteínico que tiene un papel fundamental en la regulación de la vía de señalización ERK/MAP cinasa, que intervienen en la división, diferenciación y secreción celular ${ }^{(4)}$. Las mutaciones en este gen fueron inicialmente descritas en la mitad de los pacientes con melanoma y en una pequeña proporción de pacientes con cáncer de colon; actualmente se reporta la presencia de mutaciones BRAF V600E en el $44 \%$ de los cánceres papilares de tiroides, asociándose con metástasis ganglionar a distancia y recurrencia tumoral ${ }^{(5,8)}$.

En Colombia no hay datos acerca de la presencia de mutación del gen BRAF V600E en los pacientes con cáncer de tiroi$\operatorname{des}^{(9)}$; este estudio busca describir las características clínicas e histopatológicas de los pacientes con esta patología y estudio para la mutación del gen en BRAF, diagnosticados durante los años 2014 y 2015 en la Fundación Clínica Valle del Lili.

\section{Materiales y métodos}

El estudio realizado fue observacional, descriptivo, con recolección de la información retrospectiva. De los 344 pacientes con diagnóstico de cáncer de tiroides que recibieron manejo quirúrgico en la Fundación Clínica Valle del Lili, entre el 1 de enero de 2014 y el 31 de diciembre de 2015, se logró obtener autorización de la entidad prestadora de salud para el análisis de la mutación del gen BRAF V600E en 24 pacientes. El análisis de la mutación se realizó por el grupo de patología en las biopsias, a las que se realizó la extracción del ADN en los bloques de parafina. Posteriormente se evaluaron los datos demográficos, clínicos e histopatológicos, incluyendo la estratificación TNM según la $7^{\circ}$ edición del manual de Cáncer del Comité Americano de Cáncer (AJCC) ${ }^{(10)}$ y la estratificación del riesgo de recaída según las Guías $\mathrm{ATA}^{(1)}$. Se caracterizó el tratamiento recibido, su respuesta y seguimiento, para finalmente establecer la relación de esta información con la presencia de la mutación.

\section{Análisis de la mutación BRAF}

La muestra de ADN se extrajo de los bloques de parafina de las biopsias de cada paciente por un patólogo experimentado y por medio del kit DNA Sample Preparation kit Cobas, $\left(\right.$ Roche $\left.^{\circledR}\right)$. La prueba de Mutación del gen BRAF se realizó por medio de reacción en cadena de la polimerasa en tiempo real (RT-PCR), en la Plataforma COBAS z480 (Roche ${ }^{\circledR}$ ), realizando amplificación específica del exón 15 de BRAF, analizando los resultados para la mutación V600 del BRAF por medio del Kit Cobas BRAF V600 mutation test (Roche ${ }^{\circledR}$ ). Según el protocolo recomendado por la casa comercial.

\section{Resultados}

De los 344 pacientes con diagnóstico de carcinoma diferenciado de tiroides manejados entre el 1 de enero de 2014 y el 31 de diciembre de 2015, se realizó estudio para la mutación del gen BRAF V600E a 24. En esta población, la edad promedio fue de 47 años, la mayoría menores de 45 años, con predominio en mujeres (87,5\%), (tabla 1). El tipo histológico más frecuente fue cáncer papilar de tiroides con un 95,8\%, la mayoría con presencia de la mutación (74\%).

Tabla 1. Características clínicas y patológicas de pacientes con diagnóstico de CDT con estudio para mutación BRAF V600E

\begin{tabular}{|c|c|}
\hline Característica & Frecuencia \\
\hline \multicolumn{2}{|l|}{ Edad } \\
\hline$<45$ años & $13(54 \%)$ \\
\hline$>45$ años & $11(46 \%)$ \\
\hline \multicolumn{2}{|l|}{ Sexo } \\
\hline Masculino & $3(12,5 \%)$ \\
\hline Femenino & $21(87,5 \%)$ \\
\hline \multicolumn{2}{|l|}{ Tipo histológico } \\
\hline Papilar & $23(95,8 \%)$ \\
\hline No diferenciado & $1(4,2 \%)$ \\
\hline \multicolumn{2}{|l|}{ Tamaño tumoral } \\
\hline$<1 \mathrm{~cm}$ & $6(25 \%)$ \\
\hline $1-4 \mathrm{~cm}$ & $11(45,8 \%)$ \\
\hline$>4 \mathrm{~cm}$ & $7(29,2 \%)$ \\
\hline Metástasis & $6(25 \%)$ \\
\hline No metástasis & $15(62,5 \%)$ \\
\hline Sin dato & $3(12,5 \%)$ \\
\hline \multicolumn{2}{|l|}{ Estatificación } \\
\hline I & $8(33,3 \%)$ \\
\hline II & $4(16,6 \%)$ \\
\hline III & $5(20,8 \%)$ \\
\hline IV & $7(29 \%)$ \\
\hline
\end{tabular}

En relación al patrón histológico, 23 casos correspondían a la variedad papilar, el $50 \%$ a carcinoma papilar clásico, positivos para la mutación el $66 \%$. Fueron positivos: un paciente con variante de células en tachuela, tres pacientes con variante de células altas, cinco pacientes con variedades mixtas principalmente clásico y folicular. Un paciente con variante folicular encapsulado y otro con variante de células claras fueron negativos para la mutación (tabla 2). 
Tabla 2. Relación entre las características clínicopatológicas y presencia de la mutación BRAF V600E

\begin{tabular}{|c|c|c|c|}
\hline Características & Total & $\begin{array}{c}\text { BRAF } \\
\text { positivo }\end{array}$ & $\begin{array}{c}\text { BRAF } \\
\text { negativo }\end{array}$ \\
\hline \multicolumn{4}{|l|}{ Edad } \\
\hline$<45$ años & $13(54 \%)$ & $7(54 \%)$ & $6(46 \%)$ \\
\hline$>45$ años & $11(46 \%)$ & $9(82 \%)$ & $2(18 \%)$ \\
\hline \multicolumn{4}{|l|}{ Sexo } \\
\hline Femenino & $21(87,5 \%)$ & $14(66,6 \%)$ & $7(33,3 \%)$ \\
\hline Masculino & $3(12,5 \%)$ & $2(66,6 \%)$ & $1(33,3 \%)$ \\
\hline \multicolumn{4}{|c|}{ Subtipos histológicos CA papilar } \\
\hline Clásico & $12(50 \%)$ & $8(66,6 \%)$ & $4(33,3 \%)$ \\
\hline Mixta & $5(21 \%)$ & $4(80 \%)$ & $1(20 \%)$ \\
\hline Células altas & $3(12,5 \%)$ & $3(100 \%)$ & 0 \\
\hline Tachuela & $1(4 \%)$ & 0 & $1(100 \%)$ \\
\hline Folicular & $1(4 \%)$ & 0 & $1(100 \%)$ \\
\hline Células claras & $1(4 \%)$ & 0 & $1(100 \%)$ \\
\hline \multicolumn{4}{|c|}{ Tiroiditis de Hashimoto } \\
\hline Sí & $12(50 \%)$ & $7(58 \%)$ & $5(42 \%)$ \\
\hline No & $12(50 \%)$ & $9(75 \%)$ & $3(25 \%)$ \\
\hline \multicolumn{4}{|l|}{ Multifocalidad } \\
\hline Sí & $16(66,6 \%)$ & $10(62,5 \%)$ & $6(37,5 \%)$ \\
\hline No & $8(33,3 \%)$ & $6(75 \%)$ & $2(25 \%)$ \\
\hline \multicolumn{4}{|c|}{ Invasión extratiroidea } \\
\hline Sí & $14(58 \%)$ & $11(79 \%)$ & $3(21 \%)$ \\
\hline No & $10(42 \%)$ & $5(50 \%)$ & $5(50 \%)$ \\
\hline \multicolumn{4}{|l|}{ Invasión linfática } \\
\hline Sí & $16(66,6 \%)$ & $12(75 \%)$ & $4(75 \%)$ \\
\hline No & $8(33,3 \%)$ & $4(50 \%)$ & $4(50 \%)$ \\
\hline \multicolumn{4}{|l|}{ Invasión vascular } \\
\hline Sí & $11(46 \%)$ & $8(73 \%)$ & $3(27 \%)$ \\
\hline No & $13(54 \%)$ & $8(61,5 \%)$ & $5(38,5 \%)$ \\
\hline \multicolumn{4}{|l|}{ Estadio AJCC } \\
\hline$I-I I$ & $12(50 \%)$ & $6(50 \%)$ & $6(50 \%)$ \\
\hline III-IV & $12(50 \%)$ & $10(83,3 \%)$ & $2(16,6 \%)$ \\
\hline $\begin{array}{l}\text { Total pacientes de } \\
\text { la serie }\end{array}$ & 24 & $16(66,6 \%)$ & $8(33,3 \%)$ \\
\hline
\end{tabular}

Con respecto al tamaño tumoral, 25\% tenían CDT menor a $1 \mathrm{~cm}, 66 \%$ positivos para la mutación, $45,8 \%$ entre $1-4 \mathrm{~cm}$, el $72 \%$ con la mutación y $29,2 \%$ de los casos tenía un tamaño mayor a $4 \mathrm{~cm}, 57 \%$ positivos para la mutación.

El 58\% de los casos en la patología mostraron invasión extratiroidea; de ellos, 79\% tenían expresada la mutación BRAF, $66 \%$ de los pacientes tenían invasión linfática, 75\% con la mutación, presentaron invasión vascular 46\%, tenían la mutación el 72\% (tabla 2).

De acuerdo con la clasificación TNM, 34,5\% se encontraba en estadio T1, de estos $62,5 \%$ tenían la mutación presente; T2 el 8,3\%, de los cuales $100 \%$ presentaron la mutación; T3 el $37,5 \%$, en este estadio $66 \%$ tenían la mutación, T4 el 20\%, siendo en el $60 \%$ positiva la mutación.

El 29,1\% de los casos no presentaron compromiso ganglionar, de estos el 57,1\% tenían expresada la mutación. El 33\% fueron clasificados como N1a de los cuales el 62,5\% presentaban la mutación, el 33\% se estratificaron como estadio N1b, el $75 \%$ tenían la mutación. Un paciente fue clasificado como Nxy tenía positiva la mutación BRAF V600E.

En el $62,5 \%$ de los pacientes no se documentaron metástasis a distancia, tenían la mutación el $66 \%$. El 25\% presentó metástasis pulmonares de los cuales el 50\% tenían la mutación. En tres pacientes, al momento del diagnóstico, no había información de metástasis, todos eran BRAF positivo.

Según la clasificación TNM, el 33,3\% de los pacientes correspondían al estadio I, 50\% con la mutación; $16,6 \%$ al estadio II, el 50\% presentaron la mutación; en estadio III 20,8\%, todos tenían la mutación del BRAF; en el estadio IV, 2 pacientes $(8,3 \%)$ se encontraban en estadio IVa, un paciente $(4,1 \%)$ en estadio IVb y 4 pacientes (16,6\%) en estadio IVc, de los pacientes en estadio IV, 2 eran negativos para la mutación.

Al realizar la estratificación de riesgo de recaída, según las Guías ATA 2015, tenían riesgo bajo cuatro pacientes, la mitad con la mutación, riesgo intermedio cuatro pacientes, el $75 \%$ tenían la mutación, se clasificaron en riesgo alto el $66 \%$ de los casos, el 68\% positivos para la mutación BRAF V600E (tabla 2).

\section{Discusión}

El cáncer de tiroides es una entidad altamente frecuente en nuestra población, y aunque a nivel mundial ha aumentado su prevalencia, las tasas de mortalidad no se han modificado y continúan siendo bajas. La presencia de mutaciones en el gen BRAF V600E en CDT se ha asociado en diferentes series a peor pronóstico, también a características como mayor tamaño tumoral, lesiones multifocales, metástasis a distancia y recaída, sin encontrar hasta el momento resultados concluyentes al respecto ${ }^{(11)}$.

En nuestra serie fueron positivos para la mutación BRAF V600E el $66 \%$ de los casos, similar a lo documentado en la población mundial, entre un $44 \%$ y un $75 \%{ }^{(4,5)}$. Sin embargo, se debe tener en cuenta que el estudio se realizó en un número 
bajo de pacientes, dadas las dificultades de autorización del estudio por la entidad prestadora de salud a la cual pertenecía el paciente. En relación al sexo, no se observó diferencia entre la positividad para la mutación en hombres y mujeres, lo que contrasta con lo evidenciado en el metanálisis de Li et al. en el cual se identificó una asociación entre la mutación BRAF y el sexo masculino ${ }^{(11)}$. Se encontró mayor presencia de la mutación BRAF en los pacientes mayores de 45 años (81\%), en comparación con los pacientes menores de 45 años (53\%), lo cual ha sido reportado en otras investigaciones ${ }^{(4)}$.

El cáncer papilar de tiroides es el tipo histológico más frecuentemente encontrado. En nuestra serie el $96 \%$ presentaron este tipo de cáncer, un poco por encima de lo descrito a nivel mundial, calculado aproximadamente en un $88 \%{ }^{(1)}$.

En relación a la variante de cáncer papilar encontramos que la variedad clásica, e incluso variantes de comportamiento clínico más agresivo como son la de células altas y de células en tachuela, tienen una frecuencia alta de positividad para el gen BRAF V600E, comparable a lo registrado en la literatura mundial $^{(4,7,11,12)}$.

Con respecto, al tamaño del tumor, no se encontró ninguna diferencia entre los de menor y mayor tamaño con relación a la positividad para la mutación, contrario a lo descrito en el metanálisis de Li al., al donde encuentran una clara asociación con los tumores mayores de $1 \mathrm{~cm}^{(11)}$.

En relación a características tumorales como la multicentralidad y bilateralidad del tumor, la mayoría de los pacientes positivos para la mutación no tenían estas características, contrario a lo descrito en los estudios de Li et al. ${ }^{(7)}$ y de Elisei et al., ${ }^{(10)}$ donde se encontró una asociación significativa, pero está acorde con la serie de pacientes del estudio de Sun et al. ${ }^{(4,11)}$

Los casos en los que se documentó invasión extratiroidea e invasión linfática fueron con más frecuencia positivos para la mutación, diferencia que no se encontró en los pacientes con compromiso vascular, estos datos concuerdan con lo mostrado en el estudio de Li et al. (11) $^{(1)}$

Con relación a las características del TNM, fue más frecuente el compromiso ganglionar local en los pacientes con la mutación, no se documentaron diferencias con las características tumorales ni con la presencia de metástasis, lo cual está acorde con lo encontrado en un metanálisis, donde se buscó la asociación de las caracteristicas clínicopatológicas y cáncer de tiroides ${ }^{(11)}$, diferente a lo descrito en los estudios de Sun et al. y de Liu et al. La mayor positividad para la mutación fue en pacientes clasificados como estadio III y IV, lo cual también fue descrito como una asociación en el estudio de Li et al.(11)

Al comparar los grupos, estadificándolos con el riesgo de recaída según las guías ATA, los pacientes con riesgo intermedio y alto tenían con mayor frecuencia positividad para la mutación, lo cual está acorde a lo descrito en la literatura ${ }^{(1,14)}$.

Hasta el momento, no se ha documentado ningún evento fatal relacionado con cáncer de tiroides, pero el seguimiento aún es corto, y se debe esperar la evolución de los pacientes; en recientes publicaciones la supervivencia a 5 años es mayor en los pacientes sin la mutación ${ }^{(15,16)}$.

En nuestro estudio existen limitaciones: primero, es un estudio descriptivo, por lo cual no podemos sacar evidencia fuerte de asociación; segundo, es una población pequeña a la que se le ha realizado el estudio para la mutación BRAF y, tercero, sólo tenemos datos de un único centro; sin embargo, es el primer estudio en Colombia que muestra la frecuencia de la mutación BRAF en cáncer diferenciado de tiroides, y las características clínicas e histopatológicas relacionadas con la presencia de la mutación.

\section{Conclusión}

La presencia de la mutación BRAF V600E en cáncer diferenciado de tiroides se asocia con factores de mayor agresividad tumoral, los resultados aún son contradictorios, este es el primer estudio en Colombia, que describe la relación de mutación del gen BRAF en cáncer de tiroides con hallazgos clínicos e histopatológicos, es necesario realizar más estudios que permitan refrendar estos hallazgos y que caractericen mejor la asociación, el impacto en el tratamiento y pronóstico de estos pacientes. 


\section{Referencias}

1. Haugen BR, Alexander EK, Bible KC, Doherty GM, Mandel SJ, Nikiforov YE, et al. American Thyroid Association Management Guidelines for Adult Patients with Thyroid Nodules and Differentiated Thyroid Cancer: The American Thyroid Association Guidelines Task Force on Thyroid Nodules and Differentiated Thyroid Cancer. Thyroid. 2016; 26(1):1-133.

2. Pardo CC, Ricardo. Incidencia prevalencia y mortalidad de Cancer en Colombia 2007 a 2011.

3. Dean DS, Hay ID. Prognostic indicators in differentiated thyroid carcinoma. Cancer Control. 2000;7(3):229-39.

4. Sun J, Zhang J, Lu J, Gao J, Ren X, Teng L, et al. BRAF V600E and TERT Promoter Mutations in Papillary Thyroid Carcinoma in Chinese Patients. PLoS One. 2016;11(4): e0153319.

5. Lupi C, Giannini R, Ugolini C, Proietti A, Berti P, Minuto M, et al. Association of BRAF V600E mutation with poor clinicopathological outcomes in 500 consecutive cases of papillary thyroid carcinoma. J Clin Endocrinol Metab. 2007;92(11):4085-90.

6. Kimura ET, Nikiforova MN, Zhu Z, Knauf JA, Nikiforov YE, Fagin JA. High prevalence of BRAF mutations in thyroid cancer: genetic evidence for constitutive activation of the RET/PTC-RAS-BRAF signaling pathway in papillary thyroid carcinoma. Cancer Res. 2003;63(7):1454-7.

7. Xing M. BRAF mutation in thyroid cancer. Endocr Relat Cancer 2005;12(2):245-62.

8. Xing M, Alzahrani AS, Carson KA, Shong YK, Kim TY, Viola D, et al. Association between BRAF V600E mutation and recurrence of papillary thyroid cancer. J Clin Oncol. 2015;33(1):42-50.
9. Peralta Gómez JA. Mutación BRAF V600E y su asociación con presencia de carcinoma de tiroides papilar en bacaf de pacientes con nódulo tiroideo. Biblioteca digital Universidad Nacional de Colombia 2015.

10. Edge SB BD, Compton CC, Fritz AG, Greene, FL TA. AJCC Cancer Staging Man ual. 7th ed: Springer-Verlag; 2010.

11. Li C, Lee KC, Schneider EB, Zeiger MA. BRAF V600E mutation and its association with clinicopathological features of papillary thyroid cancer: a meta-analysis. J Clin Endocrinol Metab. 2012;97(12):4559-70.

12. Asioli S, Erickson LA, Sebo TJ, Zhang J, Jin L, Thompson GB, et al. Papillary thyroid carcinoma with prominent hobnail features: a new aggressive variant of moderately differentiated papillary carcinoma. A clinicopathologic, immunohistochemical, and molecular study of eight cases. Am J Surg Pathol. 2010;34(1):44-52.

13. Jin L, Chen E, Dong S, Cai Y, Zhang X, Zhou Y, et al. BRAF and TERT promoter mutations in the aggressiveness of papillary thyroid carcinoma: a study of 65 patients. Oncotarget. 2016.

14. Tufano RP, Teixeira GV, Bishop J, Carson KA, Xing M. BRAF mutation in papillary thyroid cancer and its value in tailoring initial treatment: a systematic review and meta-analysis. Medicine (Baltimore). 2012;91(5):274-86.

15. Fraser S, Go C, Aniss A, Sidhu S, Delbridge L, Learoyd D, et al. BRAF Mutation is Associated with Decreased Disease-Free Survival in Papillary Thyroid Cancer. World J Surg. 2016

16. Mariscal SJ, Rocio. Sastre, Julia. Aso, Sonsoles. Gonzales, Ariel. Estudio de mutaciones v600e del gen BRAF en el carcinoma papilar de tiroides. 2012. 